\title{
Uso de un osciloscopio para medir la longitud de onda de un diodo láser por difracción de la luz
}

\author{
J. M. Alvarado Reyes, A. Santos Aguilar, y C. M. S. Reimer López \\ Departamento de Física, Facultad de Ciencias UNAM, \\ Av. Universidad 3000, Circuito Exterior S/N, Delegación Coyoacán, 04510 Ciudad Universitaria, Ciudad de México. \\ e-mail: jmar@ciencias.unam.mx,abrilsantos@ciencias.unam.mx; ingenieromreimer@ciencias.unam.mx
}

Received 26 April 2020; accepted 15 July 2020

\begin{abstract}
El estudio de la refracción de la luz es un fenómeno estudiado en los laboratorios de óptica de la carrera de física e ingeniería y profesiones afines. El estudio, la instrumentación y la técnica han variado muy poco desde hace décadas, por consiguiente, el error de medición sigue siendo el mismo. Entre las variables que se miden directamente durante el desarrollo y estudio de dicho fenómeno se encuentran: La distancia entre la rejilla de difracción y la pantalla donde se proyecta el fenómeno de difracción $(D)$, la longitud entre un máximo de intensidad de luz hasta donde comienza a incrementar la intensidad del segundo punto luminoso consecutivo $(y)$, y la medición indirecta del ancho de la rejilla de difracción $(a)$. De los parámetros mencionados es la distancia $y$ la que ocasiona mayor incertidumbre en el resultado dado que esta variable es dependiente del observador o experimentador. En este trabajo se presenta una propuesta innovadora donde es posible obtener este valor directamente de una gráfica, cuyos datos son adquiridos empleando un osciloscopio; los resultados obtenidos mediante el procedimiento descrito en el presente trabajo tienen una menor incertidumbre, y muestran el comportamiento de intensidades de la luz difractada.
\end{abstract}

Descriptores: Difracción; osciloscopio; longitud de onda.

The study of light refraction is a phenomenon studied in the optical laboratories of the career of physics and engineering and related professions. The study, the instrumentation and technique have changed very little for decades and, consequently, the measurement error remains the same. Among the variables that are directly measured during the development and study of said phenomenon are: the distance between the diffraction grating and the screen where the diffraction phenomenon is projected $(D)$, the length between a maximum intensity of light up to where the intensity of the second consecutive luminous point $(y)$ begins to increase, and the indirect measurement of the width of the diffraction grating $(a)$. Of the mentioned parameters, it is the distance $y$ the one that causes greater uncertainty in the result since this variable is dependent on the observer or experimenter. This work presents an innovative proposal where this value can be directly obtained from a graph whose data is acquired using an oscilloscope; the results obtained by the procedure described in this work generate less uncertainties and show the behavior of the intensity of diffracted light.

Keywords: Diffraction; oscilloscope; wavelength.

PACS: 42.25.Bs; 42.25.Fx; 01.40.gb

DOI: https://doi.org/10.31349/RevMexFisE.18.50

\section{Introducción}

\subsection{Motivación}

Los paradigmas de la enseñanza experimental han evolucionado ínfimamente; o en su defecto el uso de tecnología ha ocasionado un salto extremo. En el primer caso, los experimentos de más de un siglo [1-5] se siguen desarrollando sin cambio alguno; y los modernos emplean herramientas tales como computadoras [6], que para la mayoría de los estudiantes y profesores, son cajas negras; es decir, solo se presionan botones para obtener resultados. Son estos paradigmas de la enseñanza que motivaron a desarrollar una práctica desarrollada comúnmente en los laboratorios de óptica. En este trabajo se propone emplear un instrumento ordinario en cualquier laboratorio de enseñanza, el osciloscopio.

La difracción es un fenómeno presente en todos los tipos de ondas. Sin embargo, encontramos que en el área de enseñanza, las prácticas de laboratorio que los alumnos realizan con el fin de observar dicho fenómeno, presentan complicaciones y limitaciones que les impiden conectar sus conocimientos adquiridos en óptica geométrica y ondulatoria, electrónica, electromagnetismo e incluso sus primeros pasos en el área de la física cuántica, dejando así dudas conceptuales, que, indudablemente, se arrastran a medida que el alumno avanza en su trayectoria académica.

El diseño que describiremos a continuación, es adecuado para llevarse a cabo en el laboratorio de carreras como lo son Física e Ingeniería, debido a que el estudiante se ve en el reto de sus conocimientos elementales en óptica, electromagnetismo y electrónica, que le permitan comprobar los resultados obtenidos en el presente trabajo.

\subsection{Antecedentes (Difracción)}

En la Antigua Grecia, hace 2500 años A. C., se elaboraron teorías que intentaban explicar la naturaleza de la luz, en las que se argumentaba principalmente el modo rectilíneo en que viajaba ésta. A partir del siglo $\mathrm{XV}$, con las aportaciones de Galileo Galilei (1564-1642), el uso de instrumentos ópticos 
se generalizó y permitió la familiarización con fenómenos luminosos y su análisis. La experimentación entró en auge y la especulación fue dejándose atrás, obteniéndose así considerables resultados, hechos que en paralelo, formaron la base de los métodos de investigación científica actuales. El físico italiano Francesco María Grimaldi (1618-1663) observó por primera vez el fenómeno de la difracción, término que fue usado inicialmente por el mismo [1].

Thomas Young retoma las prácticas de Grimaldi en su investigación al realizar varios experimentos entre los cuales destacan tres para nuestro particular interés. El primero, en donde iluminó ambos bordes de un trozo delgado de cartulina observando en la pared una configuración de franjas claras y obscuras que llamó interferencia. En el segundo, experimentó con la doble rendija, que consistió en hacer pasar un haz de luz a través de una rendija con una abertura cuyo ancho es del orden de la longitud de onda del haz de luz, en consecuencia ocurre la difracción, se separa la luz y nuevamente atraviesa un obstáculo de cartulina con dos rendijas, al salir obtenemos interferencia entre ambas salidas de luz, entonces chocan cuando llegan a una pantalla donde se ven zonas claras y obscuras, imagen a la que se le conoce como patrón de interferencia. Así mismo, utilizando estas experiencias y considerando los resultados experimentales obtenidos por Isaac Newton, Thomas Young consiguió calcular las longitudes de onda de la luz roja y violeta, con $650 \mathrm{~nm}$ y 440 nm, respectivamente [1].

En este trabajo se presenta una alternativa novedosa para obtener uno de los parámetros importantes de una onda, conocido como Longitud de Onda. Para dicho fin se emplea un osciloscopio, el cual grafica directamente en su pantalla zonas oscuras y luminosas producto del fenómeno de difracción de la luz.

Visualizar el fenómeno de difracción nos permite explorar prácticas que nos anteceden y elegir la más apta para ser reproducida en el laboratorio. Algunas de las más comunes son la de Fresnel por aberturas circulares, difracción producida por una sola rendija, e interferencia producida por una doble rendija o bien conocido como el experimento de Young, entre otras. [4-5].

Para el objetivo que persigue nuestro diseño electrónico, elegimos visualizar el fenómeno por medio de una red de difracción. Cabe mencionar, que los experimentos realizados por los pioneros que estudiaron el fenómeno de interferencia y difracción forman, en gran parte, la base de las prácticas realizadas hoy en día por los estudiantes en los laboratorios de óptica. Sin embargo, es habitual que no se advierta a éstos de las dificultades más comunes del montaje experimental, así como también de las mediciones manuales y cálculos correspondientes para hallar la longitud de onda de un haz láser a partir de un patrón de difracción. Es el presente trabajo el que juega un papel importante como propuesta alternativa para la enseñanza de dicho fenómeno óptico en el laboratorio.

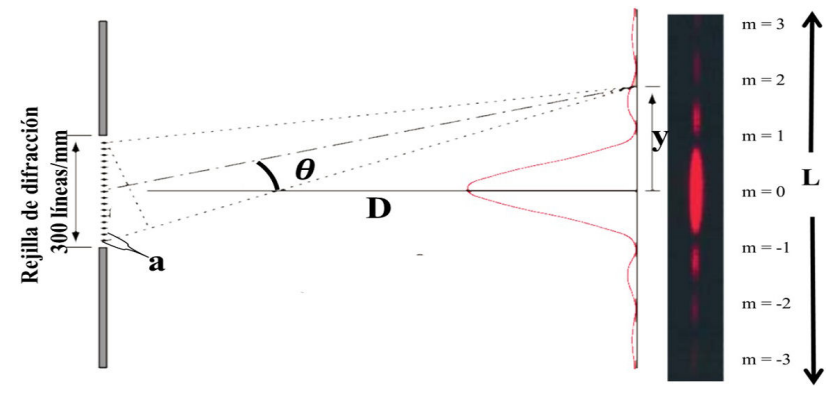

FIGURA 1. Patrón de una red de difracción.

\section{Longitud de onda en un fenómeno de difracción}

El fenómeno de difracción sucede cuando una luz incide sobre una rejilla cuyo ancho de la apertura $(a)$ sea igual o menor a la longitud de onda de la luz $(\lambda)$ [2-8]. En la Fig. 1 se representa esquemáticamente la separación entre zonas obscuras y luminosas generadas por la red de difracción. Ya que la óptica geométrica se basa en la premisa de que los rayos de luz viajan en línea recta, es posible encontrar una relación trigonométrica que nos permita obtener el ángulo $\theta$ localizado entre el máximo central $(m=0)$ y la primera franja obscura, que corresponde a la longitud $D$, de modo que la tangente del ángulo $\theta$ puede ser obtenida de la Ec. (1):

$$
\tan \theta=\frac{y}{D} \text {. }
$$

De las ecuaciones del texto elemental [7,8], el ángulo subtendido de la banda del máximo central a la primera franja obscura está dado como

$$
m \lambda=a \operatorname{sen} \theta,
$$

donde $m$ es el número de órdenes de difracción, $\lambda$ es la longitud de onda de la fuente luminosa, $a$ es el parámetro de longitud (ancho) que tiene la abertura por donde se desvía la luz. La Ec. (2) es conocida como la ecuación de la red para cualquier longitud de onda.

Si consideramos el orden $m=1$ y considerando que estamos trabajando con ángulos pequeños, tendremos la aproximación

$$
\lambda \approx a \theta .
$$

Tomando en cuenta esta consideración de ángulos pequeños es como podemos aproximar la Ec. (1), obteniendo

$$
\theta \approx \frac{y}{D},
$$

$\mathrm{y}$, sustituyendo $\theta$ en (3), obtenemos

$$
\lambda \approx a\left(\frac{y}{D}\right) .
$$

La Ec. (4) permite hallar la longitud de onda, $\lambda$, conociendo $y, a, D$ y $m$.

Es en este punto donde los estudiantes concluyen sus prácticas para hallar la longitud de onda de un láser, que genera un patrón de difracción proyectado en una pantalla. Pero, 
¿qué hay respecto a la naturaleza ondulatoria de la luz? Si observamos un patrón de difracción, vemos una diferencia de intensidades en los puntos luminosos a consecuencia de la red que fue utilizada, esto nos invita a analizarlo como un patrón de intensidades, el cual es posible visualizar en su equivalente a una magnitud de voltaje ¿Cómo? A continuación, se presenta el sistema empleado para la realización de dichas mediciones de voltaje.

\section{Sobre el sistema para medir $\lambda$}

Se diseñó y construyó un divisor de voltaje, Fig. 2, empleando una fotorresistencia como una resistencia propia del circuito, usualmente identificada como LDR del inglés lightdependent resistor, es un componente electrónico y su operación se basa en la composición del material con que se construye, en éste caso es de sulfuro de cadmio (CdS) [9], el cual tiene propiedades fotoconductivas, lo que quiere decir que su resistencia disminuye con el aumento de intensidad de la luz que incide sobre él y viceversa, Fig. 2.

Los cambios de resistencia producidos por las variaciones de intensidades de luz, detectados por el LDR, son aprovechados en un circuito electrónico conocido como divisor de voltaje, Fig. 3. Las variaciones de resistencia del LDR proporcionan, en el circuito de dicha figura, variaciones de voltaje; estos últimos son graficados en la pantalla de un osciloscopio. La ecuación del divisor de voltaje está dada por la siguiente expresión [10-11]:

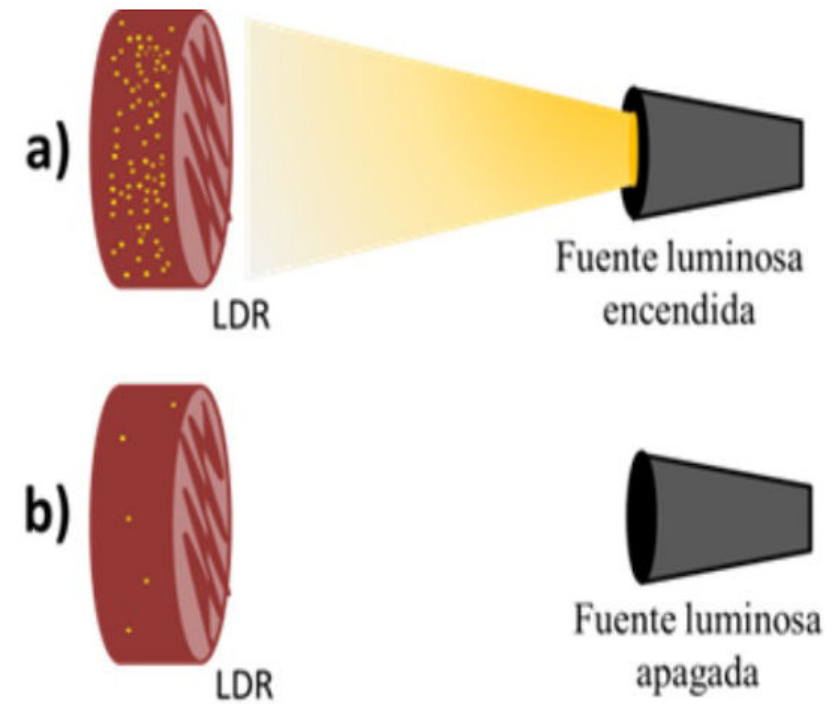

FIGURA 2. a) Situación en la que una fotorresistencia está expuesta a luz, por tanto, la energía lumínica hace que se liberen electrones, entonces el material disminuye su resistencia y aumenta su conductividad. b) Caso contrario al primero en el que la fotorresistencia no se expone a radiación y el material no libera electrones de los átomos que lo conforman.

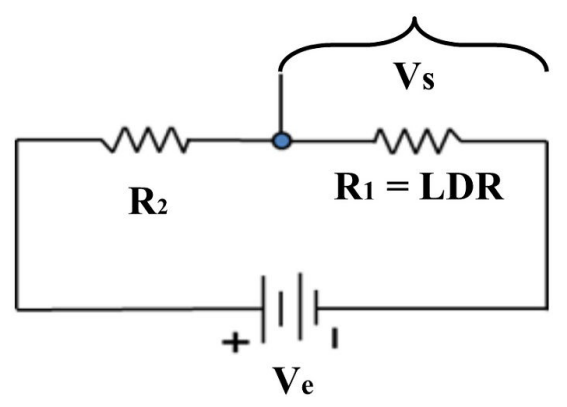

FIGURA 3. Diagrama de un de divisor de voltaje.

$$
V_{s}=\frac{V_{e} R_{1}}{R_{2}+R_{1}},
$$

donde $V_{s}$ es el voltaje de salida a través de la resistencia $R_{1}$ y $V_{e}$ es el voltaje de la pila. Las variaciones de voltaje $V_{s}$ dependerán de la variación de resistencia de la fotorresistencia $R_{1}$.

\section{Diseño experimental}

Para el montaje experimental se integró el circuito de la Fig. 3 a un riel, Fig. 4, con el fin de que este circuito pudiera desplacerse libremente de un extremo a otro sobre el riel. Para realizar este desplazamiento se usó un motor de pasos controlado con un microcontrolador, pic16F684 [12-13], Fig. 5.

El motor paso a paso, es un dispositivo electrónico que convierte la energía eléctrica en mecánica [14]. Con este dispositivo es posible controlar la velocidad, la cual es importante conocer para obtener la distancia entre dos máximos consecutivos.

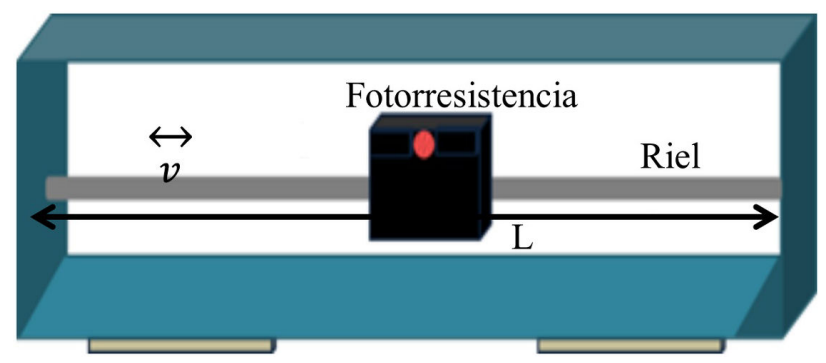

FIGURA 4. Modelo de riel donde la fotorresistencia se desplaza horizontalmente y a velocidad constante $v$.

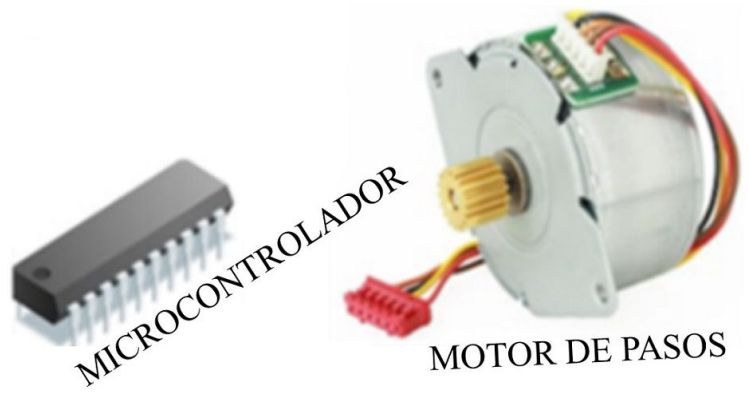

FIGURA 5. Dispositivos para el funcionamiento del motor. 


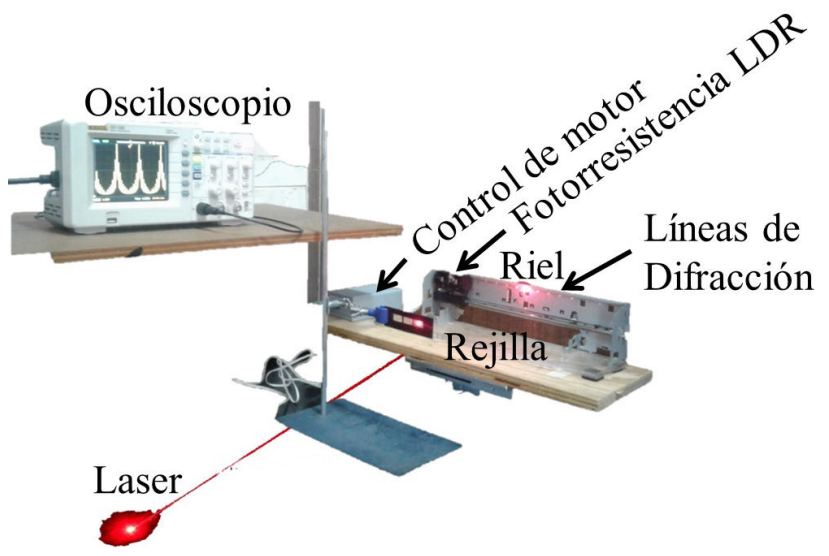

FIGURA 6. Montaje experimental empleado para medir la longitud de onda de un láser.

La detección de los puntos luminosos son detectados empleando el siguiente montaje experimental mostrado en la Fig. 6.

Dado el montaje experimental mostrado en la Fig. 6, es posible obtener en la pantalla de un osciloscopio la señal proporcionada por la detección de los puntos de luz, obtenidos por el fenómeno de difracción del haz.

\section{Resultados}

Antes de exponer los resultados obtenidos empleando el dispositivo propuesto en el presente trabajo, sería importante exponer el valor de la longitud de onda obtenido como dictan los paradigmas de la enseñanza. Para lo anterior, se presentan los valores de los siguientes parámetros medidos directamente del montaje experimental como tradicionalmente se realiza en los laboratorios de enseñanza.

\subsection{Evaluación de la longitud de onda bajo los paradig- mas de la enseñanza}

Algunos parámetros proporcionados en la Fig. 1, se miden directamente y sin ambigüedad, pero la medición de y correspondiente a la distancia entre dos máximos consecutivos luminosos o mínimos consecutivos (zonas oscuras), por lo general es una medida demasiada ambigua dependiente del experimentador.

La Ec. (4) permite hallar la longitud de onda $\lambda$ si se conocen $y, a$ y $D$ al considerar $m=1$. Podemos reescribir la Ec. (4) como

$$
\lambda \approx a\left(\frac{y}{D}\right)
$$

donde $D$ es la distancia entre la rejilla y la pantalla donde se proyecta la difracción; en nuestro caso la pantalla es un riel y el valor de $y$ que tradicionalmente se evalúa empleando la Fig. 7. Por otro lado, el ancho de la rejilla $a$ se obtiene de evaluar una sencilla regla, considerando que la rejilla de difracción empleada tiene 300 líneas/mm entonces se deduce que

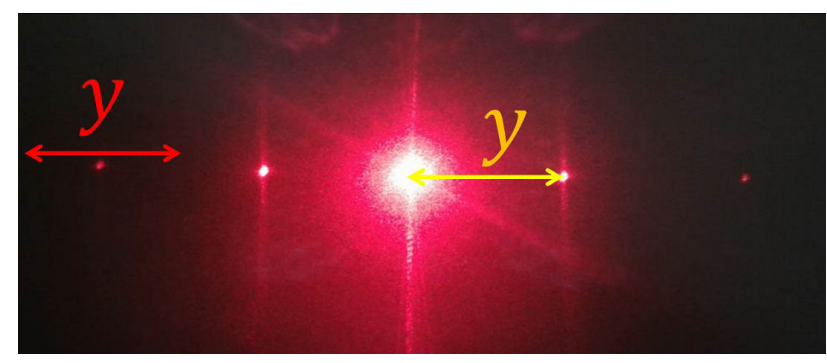

FiguRA 7. Detección de máximo y mínimos.

$$
\begin{aligned}
300 \text { líneas } & =1 \mathrm{~mm} \\
1 \text { líneas } & =x \\
\therefore \quad x & =\frac{1 \mathrm{~mm} * \text { líneas }}{300 \text { líneas }}=3.3 e^{-6} \mathrm{~m}=3.3 \mu \mathrm{m} .
\end{aligned}
$$

Es decir, el valor calculado de la rejilla es de $a=3.3 \mu \mathrm{m}$, el valor de $D=28.5 \mathrm{~cm}$ medido directamente del montaje experimental.

La medición de $y$ es la distancia cuyo origen corresponde a un máximo de intensidad de luz y finaliza en donde comienza a incrementar la intensidad del segundo punto luminoso consecutivo. El reto más importante de los estudiantes es la precisión con que se mide dicha distancia, mostrada en la Fig. 7. En ocasiones no solo la precisión recae en el instrumento de medición: regla, micrómetro o vernier, sino en la decisión de elegir el punto de máxima intensidad o el de mínima intensidad.

Durante el presente trabajo, 10 estudiantes midieron con una regla la distancia $y$, cuyos valores oscilaron entre el rango $5.45-5.65 \mathrm{~cm}$, con sus respectivas incertidumbres, donde para fines prácticos se consideran valores redondeados. Evaluando para tres valores de $y$, se obtienen los siguientes valores de $\lambda$ :

$$
\begin{aligned}
\theta_{1} & =\left(\frac{5.45}{28.5}\right)=0.19122, \\
\lambda_{1} & \approx a \theta_{1}=3.3 \mu \mathrm{m} * 0.19122, \\
\lambda_{1 \text { Tradicional }} & \approx 631.03 \mathrm{~nm} .
\end{aligned}
$$

$$
\begin{aligned}
\theta_{2} & =\left(\frac{5.55}{28.5}\right)=0.19473 \\
\lambda_{2} & \approx a \theta_{2}=3.3 \mu \mathrm{m} * 0.19473, \\
\lambda_{2 \text { _Tradicional }} & \approx 642.6 \mathrm{~nm} . \\
\theta_{3} & =\left(\frac{5.65}{28.5}\right)=0.19824, \\
\lambda_{3} & \approx a \theta_{3}=3.3 \mu \mathrm{m} * 0.19824, \\
\lambda_{3 \text { Tradicional }} & \approx 654.21 \mathrm{~nm} .
\end{aligned}
$$


El diodo láser empleado en este trabajo tiene un rango de longitudes de onda que oscila entre los 633-650 nm, de acuerdo a la compañía CrystaLaser [15]. El primer y último valor obtenido de $\lambda$ están fuera del intervalo esperado, mientras que el segundo valor se encuentra dentro. En general los paradigmas de la enseñanza hacen que se minimicen estas discrepancias dado el instrumento (regla, micrómetro o vernier) de medición empleado. Si bien lo anterior es cierto, el punto en cuestión es que la discrepancia de las $\lambda$ 's obtenidas no se debe solo al instrumento de medición empleado sino a la medición de $y$.

\subsection{Cálculo de $\lambda$ empleando un osciloscopio}

Empleando el sistema mostrado en la Fig. 6, se registran las intensidades de los diferentes puntos de difracción del láser, estas intensidades son detectadas con la ayuda de un osciloscopio, como se muestra en la Fig. 8.

La mayoría de osciloscopios digitales permiten guardar los datos correspondientes a la gráfica registrada en la pantalla, por lo que es posible analizar estos datos con un software tal como Excel o cualquier otro software científico de análisis de datos. En particular, se empleó Mat lab para graficar los datos proporcionados por el osciloscopio obteniendo la gráfica de la Fig. 9

Ya sea empleando el osciloscopio o la gráfica de datos proporcionados por el osciloscopio, es posible observar sin ambigüedad los picos de mayor y menor intensidad directamente de la gráfica.

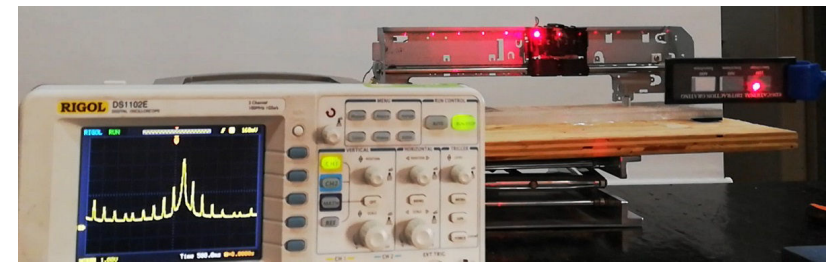

FIGURA 8. La gráfica mostrada en la pantalla del osciloscopio corresponde a las intensidades de luz de los puntos de difracción.

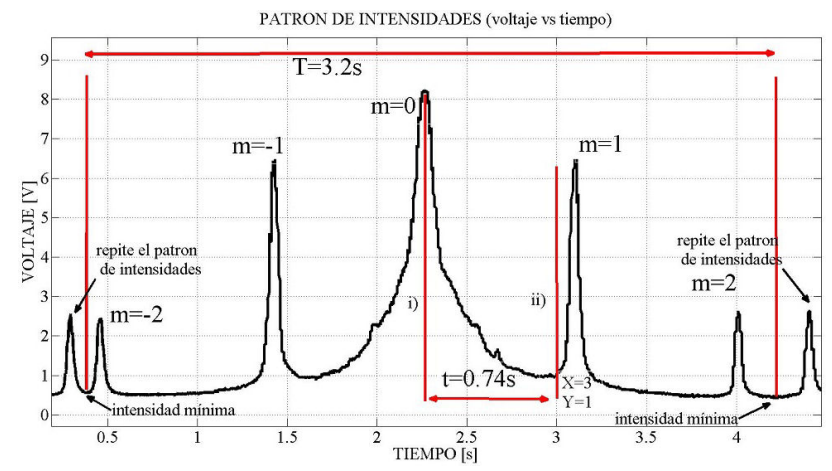

FIGURE 9. Gráfica correspondiente a la intensidad de luz difractada de los diferentes puntos de luz.
Con la gráfica adquirida por el osciloscopio se obtiene el tiempo, $t=3.2 \mathrm{~s}$, que tarda el detector LDR en viajar de un extremo a otro del riel, Fig. 8. Con este tiempo y la longitud $L=24 \mathrm{~cm}$, mostrada en la Fig. 4 y medida directamente del montaje experimental, es posible obtener la velocidad con que recorre el detector la distancia del riel,

$$
\begin{aligned}
& v_{m}=\frac{L}{t}=\frac{24 \mathrm{~cm}}{3.2 \mathrm{~s}}=7.5 \frac{\mathrm{cm}}{\mathrm{s}}, \\
& v_{m}=7.5 \frac{\mathrm{cm}}{\mathrm{s}} .
\end{aligned}
$$

Con esta velocidad podemos calcular la distancia $y$ considerando el tiempo $t=0.74 \mathrm{~s}$. Los tiempos empleados en los cálculos son obtenidos directamente de la Fig. 9.

$$
\begin{aligned}
& d=t * v_{m}=0.74 \mathrm{~s} * 7.5 \frac{\mathrm{cm}}{\mathrm{s}}, \\
& d=y=5.55 \mathrm{~cm} .
\end{aligned}
$$

Recordando que la medición de $y$ es la distancia cuyo origen corresponde a un máximo de intensidad de luz y finaliza en donde comienza a incrementar la intensidad del segundo punto luminoso consecutivo, es posible obtener estos puntos sin ambigüedad con la ayuda de la Fig. 9, o incluso directamente del osciloscopio.

$$
\begin{aligned}
\lambda_{\mathrm{Osc}} & \approx 3.3 \mu \mathrm{m} *\left(\frac{5.55}{28.5}\right) \\
& \approx 3.3 \mu \mathrm{m} * 0.19473=0.64263 \mu \mathrm{m} \\
\lambda_{\mathrm{Osc}} & =642.63 \mathrm{~nm}
\end{aligned}
$$

El valor de $\lambda_{\text {Osc }}$ no da cabida a la ambig $\tilde{A} \frac{1}{4}$ edad debido a que, ya sea con el software o el osciloscopio digital, es posible detectar los máximos y mínimos de intensidad directamente, por lo que el valor de $\lambda_{\text {Osc }}$ corresponde a la longitud de onda del diodo láser empleado, con una muy pequeña incertidumbre asociada a la resolución del osciloscopio. Sin lugar a duda, dicha incertidumbre es mucho menor a aquella obtenida en mediciones usando el ojo humano. Este hecho, es el que abre una brecha entre las mediciones manuales y el uso de instrumentos electrónicos para medir.

\section{Conclusiones}

Este trabajo no solo pretende mostrar la resolución que es posible obtener en la medición de la longitud de onda de un láser mediante el análisis de la luz difractada al incidir sobre una rejilla de difracción, sino mostrar que además es posible observar la gráfica que muestra las diferentes intensidades de los puntos de luz difractada; esta última es graficada muy pocas veces en los laboratorios de enseñanza, pero es común encontrarla en los libros de texto. Con la fotorresistencia empleada en el diseño propuesto es posible observar la gráfica de la Fig. 9, mostrada en los libros de texto. 
El método expuesto en el presente trabajo no depende del observador, sino solo de la realización y detección de las medidas hechas directamente de la gráfica proporcionada por el osciloscopio.

El sistema propuesto proporciona muy poca incertidumbre en el valor de la longitud de onda debido a que se analizan datos correspondientes a los picos de máxima intensidad y baja intensidad de la luz difractada. Estos últimos son medibles sin ambigüedad sobre la gráfica proporcionada por el osciloscopio.

El valor de $\lambda_{\text {Osc }}$ obtenido en este trabajo, empleando un osciloscopio, muestra con claridad que el error usual obtenido en los laboratorios de enseñanza experimental es debido al observador, ocasionado por la intensa luminosidad del punto central y la selección de la zona de poca luminosi- dad. Estos puntos son una selección ambigua, situación por la cual el presente trabajo tiene una gran importancia.

El lector puede notar que se proporcionó muy poca información sobre el diseño y construcción del sistema electrónico propuesto en este trabajo. La razón de esto es que el presente trabajo se enfocó a la aplicación del sistema; no obstante, la información del circuito y la programación puede ser consultada en la página web [16]. Así mismo se puede solicitar información a los correos de los autores.

\section{Agradecimientos}

Este trabajo es un producto apoyado por el proyecto PAPIME PE110216.
1. S. Bravo y M. Pesa, El fenómeno de difracción en la Historia de la óptica y en los libros de texto: reflexiones sobre sus dificultades de aprendizaje. Investig. Ensino Ciênc. 20 (2015) 76. https://doi.org/10.22600/ 1518-8795.ienci2016v20n2p76.

2. D. Blanco Laserna, Luz y ondas. Huygens: la luz como propagación ondulatoria. Arbor, 191 (2015) a263. http://dx. doi.org/10.3989/arbor.2015.775n5002

3. G. Camacho Basilio, G. Aguilar Soto, R. Carrada Legaria. Manual de proyectos en óptica. (sin publicar, 2004).

4. C. D. Molina, A. F. Calvo, y L. E. Pamplona, Desarrollo de un módulo experimental de difracción de la luz para un laboratorio de física controlado de forma remota, Prospectiva 15 (2017) 100. https://doi.org/10.15665/rp.v15i1.744

5. L. Garrigos Oltra, Utilización del Láser en Experiencias por difracción: Cálculo de la Longitud de Onda de una radiación Luminosa, Enseñ. Cienc., 1 (1983) 46. https://www.raco.cat/index.php/ensenanza/ article/viewFile/50614/92621

6. O. Cortes, A. Jimy, O. Chaves, J. André y. J. A. Mendoza, Estudio de la difracción de Fraunhofer de una ranura simple mediante tratamiento digital de imágenes. Sci. Tech. 18 (2013) 293. http: //www.redalyc.org/pdf/849/ $84927487043 . \mathrm{pdf}$

7. P. A. Tipler, Física para la ciencias y tecnología 4 ta ed. (Reverté, Barcelona, 2001), Vol. 2, p. 1083.

8. H. Benson, University Physics (John Wiley, New Jersey, 1991), p. 842 .

9. http://roble.pntic.mec.es/jlop0164/ archivos/LDR-termistor.pdf
10. H. Robbins, Wilhelm C. Miller. Análisis de Circuitos Teoría y Práctica, 4ta ed. (Cengage Learning, MÃ@)xico, 2008), p. 126. http://prof.usb.ve/mirodriguez/ circuito_electrico_i/libro.pdf

11. T. L. Floyd, Principios de circuitos eléctricos, 8va ed. (Pearson, México, 2007). http://media.espora. org/mgoblin_media/media_entries/1455/ Principios_de_circuitos_electricos.pdf

12. E. A. García Breijo, Compilador C CCS y simulador Proteus para microcontroladores PIC, 2da ed. (Marcombo, Barcelona, 2009). https://tecnologialsb.files. wordpress.com/2017/05/compilador-c-ccs-y -simulador-proteus-para-microcontroladores -pic.pdf

13. J. M. Angulo Usategui e I. Angulo Martínez, Microcontroladores PIC, 3ra ed. (McGraw-Hill/Interamericana, Madrid, 2003).

14. SureStep, Manual No. STP-SYS-MSP, 2005. https:// cdn.automationdirect.com/static/manuals/ surestepmanualsp/surestepmanualsp.pdf

15. http://www.crystalaser.com/ new/redlaser.html?gclid= CjwKCAjw-YT1BRAFEiwAd2WRt lKb5zVGqYRe7 ?gclid=CjwKCAjw-YT1BRAFEiwAd2WRt lKb5 zVGq YRe 7bpvHb4 rpqz15bUd6ou0Kn3GVRb. 1NZ6KyhvjoupsRoCoWkQAvD_BwE

16. http://academicos.fciencias.unam.mx/jmar/ 